\title{
TWISTED ALEXANDER POLYNOMIALS AND CHARACTER VARIETIES OF 2-BRIDGE KNOT GROUPS
}

\author{
TAEHEE KIM AND TAKAYUKI MORIFUJI
}

\begin{abstract}
We study the twisted Alexander polynomial from the viewpoint of the $S L(2, \mathbb{C})$-character variety of nonabelian representations of a knot group. It is known that if a knot is fibered, then the twisted Alexander polynomials associated with nonabelian $S L(2, \mathbb{C})$-representations are all monic. In this paper, we show that the converse holds for 2-bridge knots. Furthermore we show that for a 2-bridge knot there exists a curve component in the $S L(2, \mathbb{C})$ character variety such that if the knot is not fibered then there are only finitely many characters in the component for which the associated twisted Alexander polynomials are monic. We also show that for a 2-bridge knot of genus $g$, in the above curve component for all but finitely many characters the associated twisted Alexander polynomials have degree $4 g-2$.
\end{abstract}

\section{INTRODUCTION}

In 22, Lin introduced the twisted Alexander polynomial for knots in the 3-sphere by using regular Seifert surfaces, while Wada [31] defined it for finitely presentable groups, which include link groups. It is a generalization of the classical Alexander polynomial and has many applications to knot theory. A notable application is to distinguish two mutant knots with the trivial Alexander polynomial [31. The twisted Alexander polynomial can be regarded as the Reidemeister torsion and this fact leads to its symmetry [18. It is also useful to the problems on inversion and concordance of knots [17. Furthermore classical results about the Alexander polynomial of fibered knots are extended to the twisted case [3, 9, 10, 12]. In particular, in [10] Friedl and Vidussi showed that the twisted Alexander polynomials corresponding to all finite representations detect fibered 3-manifolds. For literatures on the twisted Alexander polynomial and other related topics, refer to the survey paper by Friedl and Vidussi [1].

The purpose of this paper is to consider another approach to the fibering problem for knots. That is, we study the problem from the viewpoint of the $S L(2, \mathbb{C})$ character variety of a knot group. The approach of using the character variety to 3-manifold problems was introduced by Culler and Shalen [5], and since then, many deep results on 3-manifolds have been obtained using the character variety. Roughly speaking, many topological properties of a 3-manifold are encoded in the character variety of the 3-manifold group, and therefore it is interesting to study basic properties of the character variety.

In general, for a given knot each coefficient of the twisted Alexander polynomial of the knot defines a complex valued function on the $S L(2, \mathbb{C})$-representation variety of the knot group. If the coefficient of the highest degree term is the constant

2000 Mathematics Subject Classification. Primary 57M27, Secondary 57M05, 57M25.

Key words and phrases. Twisted Alexander polynomial, character variety, 2-bridge knot. 
function 1, then we call such a representation (and its character) monic. In this point of view, it is known that every nonabelian $S L(2, \mathbb{C})$-representation of a fibered knot is monic 12. One can ask if the converse holds, but the answer is still unknown (cf. 10]). In this paper, we give a partial answer that the converse holds for 2bridge knots: for a 2-bridge knot, if every nonabelian $S L(2, \mathbb{C})$-representation of the knot is monic, then the knot is fibered (Theorem 4.1). In fact, we show a much stronger theorem that for a nonfibered 2-bridge knot, there exists a curve component in the character variety of nonabelian representations of the knot in which there are only finitely many monic characters (Theorem 4.2). This extends the result for twist knots of the second author in 24. We also give a sufficient condition based on the Alexander polynomial for a knot to have only finitely many monic characters (Theorem 4.5). Moreover we give an upper bound of the number of monic characters for a family of certain 2-bridge knots which contains twist knots (Theorem 5.1). Although fiberedness of a 2-bridge knot is detected by the Alexander polynomial since 2-bridge knots are alternating, these results give new fibering criteria for 2-bridge knots and lead us to a conjecture that Theorem 4.2 can be extended for a general nonfibered knot (see Conjecture 6.4 and Remark 6.5).

In addition to studying the fibering problem, we also investigate detecting the genus of a knot using the twisted Alexander polynomial. Recall that a 2-bridge knot is alternating, hence its classical Alexander polynomial is of degree $2 g$ where $g$ denotes the genus of the knot. It is also known that if a knot of genus $g$ is fibered, then the twisted Alexander polynomial associated with any nonabelian $S L(2, \mathbb{C})$ representation is of degree $4 g-2$ (see [19]). In this direction, we show that for a (possibly nonfibered) 2-bridge knot of genus $g$, there exists a curve component in the character variety of nonabelian representations of the knot such that for all but finitely many characters in the component the associated twisted Alexander polynomials have degree $4 g-2$ (Theorem 4.3).

This paper is organized as follows. In the next section, we review some basic materials for the character variety, in particular, the representation polynomial of a 2-bridge knot group due to Riley [30. In Section 3, we quickly review the definition of the twisted Alexander polynomials associated with $S L(2, \mathbb{C})$-representations. In Section 4, we show finiteness of monic characters in a curve component of the character variety of nonabelian representations of a nonfibered 2-bridge knot. We also show the genus of a 2-bridge knot is detected by the twisted Alexander polynomial and the character variety of the knot group. Section 5 is devoted to the calculation of an upper bound of the number of monic characters for a family of 2-bridge knots. In the last section, we give some remarks and state conjectures on the fibering problem from the viewpoint of $S L(2, \mathbb{C})$-representations of a knot group.

\section{Character varieties}

In this section we review the theory of the character variety of a knot group that will be needed for our purpose. See [5, 21, 30] for details.

Let $G$ be a finitely generated group. Define $R(G)=\operatorname{Hom}(G, S L(2, \mathbb{C}))$ to be the set of representations of $G$ into $S L(2, \mathbb{C})$. It is known that $R(G)$ is a complex affine algebraic set. We call it the $S L(2, \mathbb{C})$-representation variety of $G$, though it might be a union of a finite number of irreducible algebraic varieties in the sense of 
algebraic geometry. The isomorphism class of this variety does not depend on the choice of generators.

The group $S L(2, \mathbb{C})$ acts on $R(G)$ by conjugation. Let $\hat{R}(G)$ denote the set of orbits. Two representations $\rho, \rho^{\prime} \in R(G)$ are called conjugate if they lie in the same orbit. The algebro-geometric quotient of $R(G)$ under this conjugate action is called the $S L(2, \mathbb{C})$-character variety of $G$, which is denoted by $X(G)$. The character of a representation $\rho$ is a map $\chi_{\rho}: G \rightarrow \mathbb{C}$ defined by $\chi_{\rho}(\gamma)=\operatorname{tr}(\rho(\gamma))$ for $\gamma \in G$. There is a bijection between $X(G)$ and the set of characters of $G$. Namely, we have a canonical identification $X(G)=\left\{\chi_{\rho} \mid \rho \in R(G)\right\}$.

A representation $\rho: G \rightarrow S L(2, \mathbb{C})$ is said to be abelian if $\rho(G)$ is an abelian subgroup of $S L(2, \mathbb{C})$. A representation $\rho$ is called reducible if there exists a proper invariant subspace in $\mathbb{C}^{2}$. This is equivalent to saying that $\rho$ can be conjugated to a representation by upper triangular matrices. It is easy to see that every abelian representation is reducible, but the converse does not hold. Namely there is a reducible nonabelian representation in general. When $\rho$ is not reducible, it is called irreducible. If $\rho, \rho^{\prime} \in R(G)$ have the same character $\chi_{\rho}=\chi_{\rho^{\prime}}$ and $\rho$ is irreducible, then $\rho$ is conjugate to $\rho^{\prime}$ (see [5, Proposition 1.5.2]).

Let $R^{\mathrm{irr}}(G)$ denote the subset of irreducible representations of $G$ and $X^{\mathrm{irr}}(G)$ denote its image under the map $t: R(G) \rightarrow X(G)$ given by $t(\rho)=\chi_{\rho}$. Similary, we write $X^{\mathrm{nab}}(G)$ for the image of $R^{\mathrm{nab}}(G)$, the set of nonabelian $S L(2, \mathbb{C})$ representations, by $t$.

If $G$ is a knot group $G(K)$, namely the fundamental group of the exterior $E(K)$ of a knot $K$ in the 3-sphere $S^{3}$, we denote $R(G(K))$ and $X(G(K))$ by $R(K)$ and $X(K)$ for simplicity. We also use the notations $X^{\text {irr }}(K), X^{\text {nab }}(K)$ and so on.

Now we quickly review how to describe the nonabelian part of the $S L(2, \mathbb{C})$ character variety of a 2-bridge knot group (see [21, 30] for details). Let $K=K(\alpha, \beta)$ be a 2-bridge knot corresponding to a pair of relatively prime odd integers $(\alpha, \beta)$ with $-\alpha<\beta<\alpha$. The knots $K(\alpha, \beta)$ and $K\left(\alpha^{\prime}, \beta^{\prime}\right)$ have the same type if and only if $\alpha=\alpha^{\prime}$ and $\beta \equiv \beta^{\prime}$ or $\beta \beta^{\prime} \equiv 1 \bmod \alpha$. The knot group of $K$ has a presentation

$$
G(K)=\langle a, b \mid w a=b w\rangle, \quad w=a^{\epsilon_{1}} b^{\epsilon_{2}} \cdots a^{\epsilon_{\alpha-2}} b^{\epsilon_{\alpha-1}}
$$

where $\epsilon_{i}=(-1)^{\left[\frac{\beta}{\alpha} i\right]}$ and $[u]$ denotes the greatest integer less than or equal to $u \in \mathbb{R}$. It follows that $\epsilon_{i}=\epsilon_{\alpha-i}$ holds for any $i$.

The above presentation for $G(K)$ is not unique, but the existence of at least one such presentation follows from Wirtinger's algorithm applied to Schubert's canonical 2-bridge diagram of $K(\alpha, \beta)$. The generators $a$ and $b$ come from the two bridges (overpasses) and represent the meridian up to conjugation.

We consider the matrices $C=\left(\begin{array}{cc}s & 1 \\ 0 & s^{-1}\end{array}\right)$ and $D=\left(\begin{array}{cc}s & 0 \\ 2-y & s^{-1}\end{array}\right)$, where $s \neq 0, y \in \mathbb{C}$. Since $a$ and $b$ are conjugate in the group $G(K)$, for a nonabelian representation $\rho: G(K) \rightarrow S L(2, \mathbb{C}), \rho(a)$ and $\rho(b)$ have the same trace. Moreover, taking conjugation if necessary, we can assume $\rho(a)=C$ and $\rho(b)=D$ without loss of generality. Here the entry $2-y$ is chosen so that $C D^{-1}$ has trace $y$ (see [23]). Under the setting above, we have the following.

Proposition 2.1. 30, Theorem 1] The assignment $\rho(a)=C, \rho(b)=D$ defines a nonabelian representation of $G(K)$ if and only if the pair $(s, y)$ satisfies the equation

$$
w^{11}+\left(s^{-1}-s\right) w^{12}=0,
$$


where $W=\rho(w)=\left(w^{i j}\right)$. Conversely, every nonabelian representation of $G(K)$ is conjugate to a representation satisfying the above equation.

We now define the polynomial $\phi(s, y)$ via

$$
\phi(s, y)=w^{11}+\left(s^{-1}-s\right) w^{12} \in \mathbb{Z}\left[s^{ \pm 1}, y\right]
$$

and call it the Riley polynomial of a 2-bridge knot $K$. That is, the Riley polynomial gives a defining equation of the nonabelian part $\hat{R}^{\text {nab }}(K)$ of conjugacy classes of $S L(2, \mathbb{C})$-representations. We also remark here that $\phi(s, y)$ might be a reducible polynomial even over $\mathbb{Z}\left[s^{ \pm 1}, y\right]$.

Example 2.2. Let $K$ be the knot $K(15,11)=7_{4}$ with the Alexander polynomial $\Delta_{K}(t)=4-7 t+4 t^{2}$. The knot group has a presentation

$$
G(K)=\langle a, b \mid w a=b w\rangle, \quad w=\left(\left(b a^{-1}\right)^{2}\left(b^{-1} a\right)^{2}\right)^{2} .
$$

A direct calculation shows that the Riley polynomial $\phi(s, y)$ has the factorization

$$
\phi(s, y)=\phi_{1}(s, y) \phi_{2}(s, y) \in \mathbb{Z}\left[s^{ \pm 1}, y\right],
$$

where

$$
\begin{aligned}
& \phi_{1}(s, y)=1+\left(s^{-2}+s^{2}\right) y^{2}-y^{3}, \\
& \phi_{2}(s, y)=1-2\left(s^{-2}+s^{2}\right) y+\left(3 s^{-2}+2+3 s^{2}\right) y^{2}-\left(s^{-2}+3+s^{2}\right) y^{3}+y^{4} .
\end{aligned}
$$

Putting $y=2, \phi_{1}(s, 2)=4 s^{-2}-7+4 s^{2}=s^{-2} \Delta_{K}\left(s^{2}\right)=0$ gives reducible nonabelian representations (see Proposition 2.4 below). On the other hand, $\phi_{2}(s, y)=0$ contains no reducible representation.

For $\gamma \in G(K)$, let $t_{\gamma}$ be a function $t_{\gamma}: R(K) \rightarrow \mathbb{C}$ defined by $t_{\gamma}(\rho)=\operatorname{tr}(\rho(\gamma))$. Then as a coordinate of $X^{\mathrm{nab}}(K)$ for a 2-bridge knot $K$, we can take $\left(t_{a}, t_{a b^{-1}}\right)$. Namely, $X^{\mathrm{nab}}(K)$ can be identified with the image of $R^{\mathrm{nab}}(K)$ under the map $\left(t_{a}, t_{a b^{-1}}\right): R(K) \rightarrow \mathbb{C}^{2}$ (see [5, Proposition 1.4.1] and [23, Section 2]). Of course, this map factors through $\hat{R}(K)$. If $\phi$ is viewed as a polynomial in $x=s+s^{-1}$ and $y$, then $X^{\mathrm{nab}}(K)$ is given in $\mathbb{C}^{2}$ by $\phi(x, y)=0$ (see [23, Proposition 2.2]).

Remark 2.3. It is known that for a 2-bridge knot $K$ the Zariski closure $\overline{X^{\text {irr }}(K)}$ is exactly the nonabelian part of the character variety $X^{\text {nab }}(K)$ (see [21]). More precisely, except finitely many cases, a nonabelian representation is irreducible.

We end this section with the following useful proposition.

Proposition 2.4 (Burde 2, de Rham [7). Let $\eta_{0}: G(K) \rightarrow S L(2, \mathbb{C})$ be an abelian representation of a knot $K$ given by $\eta_{0}(\mu)=\left(\begin{array}{cc}\lambda & 0 \\ 0 & \lambda^{-1}\end{array}\right)$, where $\mu$ is the meridian of $K$ and $\lambda \neq 0 \in \mathbb{C}$. Then there is a reducible nonabelian representation $\rho: G(K) \rightarrow S L(2, \mathbb{C})$ so that $\chi_{\rho}=\chi_{\eta_{0}}$ if and only if $\Delta_{K}\left(\lambda^{2}\right)=0$.

\section{Twisted Alexander polynomials}

For a knot group $G(K)=\pi_{1}(E(K))$, we choose and fix a Wirtinger presentation

$$
G(K)=\left\langle\gamma_{1}, \ldots, \gamma_{k} \mid r_{1}, \ldots, r_{k-1}\right\rangle .
$$

Then the abelianization homomorphism

$$
\alpha: G(K) \rightarrow H_{1}(E(K), \mathbb{Z}) \cong \mathbb{Z}=\langle t\rangle
$$


is given by $\alpha\left(\gamma_{1}\right)=\cdots=\alpha\left(\gamma_{k}\right)=t$. Here we specify a generator $t$ of $H_{1}(E(K), \mathbb{Z})$ and denote the sum in $\mathbb{Z}$ multiplicatively. In this paper we consider just a linear representation $\rho: G(K) \rightarrow G L(2, \mathbb{C})$.

These maps $\rho$ and $\alpha$ naturally induce two ring homomorphisms $\tilde{\rho}: \mathbb{Z}[G(K)] \rightarrow$ $M(2, \mathbb{C})$ and $\tilde{\alpha}: \mathbb{Z}[G(K)] \rightarrow \mathbb{Z}\left[t^{ \pm 1}\right]$, where $\mathbb{Z}[G(K)]$ is the group ring of $G(K)$ and $M(2, \mathbb{C})$ is the matrix algebra of degree 2 over $\mathbb{C}$. Then $\tilde{\rho} \otimes \tilde{\alpha}$ defines a ring homomorphism $\mathbb{Z}[G(K)] \rightarrow M\left(2, \mathbb{C}\left[t^{ \pm 1}\right]\right)$. Let $F_{k}$ denote the free group on generators $\gamma_{1}, \ldots, \gamma_{k}$ and

$$
\Phi: \mathbb{Z}\left[F_{k}\right] \rightarrow M\left(2, \mathbb{C}\left[t^{ \pm 1}\right]\right)
$$

the composition of the surjection $p: \mathbb{Z}\left[F_{k}\right] \rightarrow \mathbb{Z}[G(K)]$ induced by the presentation of $G(K)$ and the map $\tilde{\rho} \otimes \tilde{\alpha}: \mathbb{Z}[G(K)] \rightarrow M\left(2, \mathbb{C}\left[t^{ \pm 1}\right]\right)$.

Let us consider the $(k-1) \times k$ matrix $M$ whose $(i, j)$-entry is the $2 \times 2$ matrix

$$
\Phi\left(\frac{\partial r_{i}}{\partial \gamma_{j}}\right) \in M\left(2, \mathbb{C}\left[t^{ \pm 1}\right]\right),
$$

where $\frac{\partial}{\partial \gamma}$ denotes the Fox differential. This matrix $M$ is called the Alexander matrix of $G(K)$ associated with the representation $\rho$.

For $1 \leq j \leq k$, let us denote by $M_{j}$ the $(k-1) \times(k-1)$ matrix obtained from $M$ by removing the $j$ th column. We regard $M_{j}$ as a $2(k-1) \times 2(k-1)$ matrix with coefficients in $\mathbb{C}\left[t^{ \pm 1}\right]$.

Then Wada's twisted Alexander polynomial of a knot $K$ associated with a representation $\rho: G(K) \rightarrow G L(2, \mathbb{C})$ is defined to be the rational function

$$
\Delta_{K, \rho}(t)=\frac{\operatorname{det} M_{j}}{\operatorname{det} \Phi\left(1-\gamma_{j}\right)}
$$

and well-defined up to multiplication by $\varepsilon t^{2 i}\left(\varepsilon \in \mathbb{C}^{*}, i \in \mathbb{Z}\right)$. Moreover for the case of a special linear representation $\rho: G(K) \rightarrow S L(2, \mathbb{C}), \Delta_{K, \rho}(t)$ is well-defined up to multiplication by $t^{2 i}(i \in \mathbb{Z})$. In this paper mostly we use a representation $\rho: G(K) \rightarrow S L(2, \mathbb{C})$.

Remark 3.1. If $\rho$ is conjugate to $\rho^{\prime}$ in $G L(2, \mathbb{C})$, then $\Delta_{K, \rho}(t)=\Delta_{K, \rho^{\prime}}(t)$ holds (see 31, Section 3]). Moreover $\Delta_{K, \rho}(t)$ has the following properties. In this paper, a (Laurent) polynomial $f(t)=c_{m} t^{m}+c_{m-1} t^{m-1}+\cdots+c_{n+1} t^{n+1}+c_{n} t^{n} \in \mathbb{C}\left[t^{ \pm 1}\right]$ is called monic if the coefficient $c_{m}$ is just 1 (not \pm 1 ). Let $\rho: G(K) \rightarrow S L(2, \mathbb{C})$ be a nonabelian representation.

(i) The twisted Alexander polynomial $\Delta_{K, \rho}(t)$ is always a polynomial for any knot $K$ (see [19, Theorem 3.1]), and it is reciprocal, i.e., $\Delta_{K, \rho}(t)=$ $t^{i} \Delta_{K, \rho}\left(t^{-1}\right)$ for some $i \in \mathbb{Z}$ (see [14, Corollary 3.5]).

(ii) If $K$ is a fibered knot of genus $g$, namely the exterior $E(K)$ has the structure of a surface bundle over the circle, then $\Delta_{K, \rho}(t)$ becomes a monic polynomial of degree $4 g-2$ (see [12, Theorem 3.1] and [19, Theorem 3.2]).

(iii) If $K$ is a knot of genus $g$, then $\operatorname{deg}\left(\Delta_{K, \rho}(t)\right) \leq 4 g-2$ (see 9. Theorem $1.1])$.

(iv) If $\rho$ is a reducible nonabelian representation, then up to conjugation, $\rho\left(\gamma_{i}\right)=\left(\begin{array}{cc}\lambda & \nu_{i} \\ 0 & \lambda^{-1}\end{array}\right)$ for each $i$ where $\lambda \neq 0, \nu_{i} \in \mathbb{C}$ and

$$
\Delta_{K, \rho}(t)=\frac{\Delta_{K}(\lambda t) \Delta_{K}\left(\lambda^{-1} t\right)}{(t-\lambda)\left(t-\lambda^{-1}\right)}
$$


(See the proof of [19, Theorem 3.1].) In particular, $\Delta_{K, \rho}(t)$ is a polynomial of degree $2 \operatorname{deg}\left(\Delta_{K}(t)\right)-2$.

Example 3.2. Let $\eta: G(K) \rightarrow S L(2, \mathbb{C})$ be an abelian representation defined by the correspondence $\gamma_{i} \mapsto\left(\begin{array}{cc}\lambda & \nu \\ 0 & \lambda^{-1}\end{array}\right)$ for any $i$, where $\lambda \neq 0, \nu \in \mathbb{C}$. Then

$$
\Delta_{K, \eta}(t)=\frac{\Delta_{K}(\lambda t) \Delta_{K}\left(\lambda^{-1} t\right)}{(t-\lambda)\left(t-\lambda^{-1}\right)}
$$

but it is not a polynomial in general.

Let $\rho: G(K) \rightarrow S L(2, \mathbb{C})$ be a nonabelian representation of a knot $K$. Then each coefficient of $\Delta_{K, \rho}(t)$ defines a complex valued function on the representation variety $R^{\mathrm{nab}}(K)$, the orbit space $\hat{R}^{\mathrm{nab}}(K)$ and also on the character variety $X^{\mathrm{nab}}(K)$. In fact, if $\rho$ is a reducible nonabelian representation, there is an abelian representation $\eta_{0}$ so that $\chi_{\eta_{0}}=\chi_{\rho}$ (see Proposition 2.4). Of course, $\rho$ and $\eta_{0}$ are not conjugate, but $\Delta_{K, \rho}(t)=\Delta_{K, \eta_{0}}(t)$ holds (see Remark 3.1 (iv) and Example 3.2). Therefore each coefficient of $\Delta_{K, \rho}(t)$ can be considered as a function on $X^{\text {nab }}(K)$. Also we define the twisted Alexander polynomial associated with $\chi \in X^{\mathrm{nab}}(K)$ to be $\Delta_{K, \rho}(t)$ where $\chi=\chi_{\rho}$, and denote it by $\Delta_{K, \chi}(t)$. In particular, for a 2-bridge knot $K$, since $X^{\mathrm{nab}}(K)$ is given in $\mathbb{C}^{2}$ by the equation $\phi(x, y)=0$ where $x=s+s^{-1}$, each coefficient of $\Delta_{K, \rho}(t)$ can be considered as a function of $s$ and $y$ or a function of $x$ and $y$.

It should be noted that the notion of monic polynomial makes sense for $\Delta_{K, \rho}(t)$ by the indeterminacy of $\Delta_{K, \rho}(t)$ and Remark 3.1 (i). We say a nonabelian representation $\rho: G(K) \rightarrow S L(2, \mathbb{C})$ is monic if the twisted Alexander polynomial $\Delta_{K, \rho}(t)$ associated with $\rho$ is a monic polynomial. Similary we call $\chi \in X^{\mathrm{nab}}(K)$ monic if $\Delta_{K, \chi}(t)$ is a monic polynomial. For a fibered knot $K$ in $S^{3}$, all the nonabelian $S L(2, \mathbb{C})$-representations are monic [12. In other words, the coefficient of the highest degree term of $\Delta_{K, \rho}(t)$ is a constant function 1 on the whole $X^{\mathrm{nab}}(K)$.

\section{Finiteness theOREMS FOR 2-BRIDGe KnOts}

Below we show that for a 2-bridge knot $K$ in $S^{3}, X^{\mathrm{nab}}(K)$ detects if $K$ is fibered. That is, the converse of Remark 3.1 (ii) holds for 2-bridge knots.

Theorem 4.1. A 2-bridge knot $K$ is fibered if and only if $\Delta_{K, \rho}(t)$ is monic for any nonabelian representation $\rho: G(K) \rightarrow S L(2, \mathbb{C})$.

Proof. The 'if' part was proven in [12. Now assume that $K$ is a nonfibered 2bridge knot. We choose and fix a Wirtinger presentation $G(K)=\langle a, b \mid w a=b w\rangle$ as we did in Section 2 . Let $\rho: G(K) \rightarrow S L(2, \mathbb{C})$ be an arbitrary nonabelian representation. We will show that the coefficient of the highest degree term of $\Delta_{K, \rho}(t)$ is not identically one on $X^{\mathrm{nab}}(K)$.

By taking conjugations if necessary, we may assume that

$$
\rho(a)=\left(\begin{array}{cc}
s & 1 \\
0 & s^{-1}
\end{array}\right), \quad \rho(b)=\left(\begin{array}{cc}
s & 0 \\
2-y & s^{-1}
\end{array}\right) .
$$

Suppose that the top coefficient of $\Delta_{K, \rho}(t)$ is identically one on $X^{\text {nab }}(K)$. Namely, we assume that

$$
\Delta_{K, \rho}(t)=t^{m}+f_{1}(s, y) t^{m-1}+f_{2}(s, y) t^{m-2}+\cdots
$$


holds. Since the Alexander polynomial of a 2-bridge knot is not trivial, by Proposition 2.4 there exists a reducible nonabelian representation of $K$. Since $\rho$ becomes reducible exactly when $y=2$ (see [5, Proposition 1.5.5]), it means that there exists $s_{0} \in \mathbb{C}^{*}$ such that $\phi\left(s_{0}+s_{0}^{-1}, 2\right)=0$ viewing $\phi$ as a polynomial of $x=s+s^{-1}$ and $y$, and therefore $\rho$ with $s=s_{0}$ and $y=2$ becomes a reducible nonabelian representation, say $\rho^{\prime}$. For $\rho^{\prime}$, by Remark 3.1 (iv) we obtain that $\Delta_{K, \rho^{\prime}}(t)=\operatorname{det} M /(t-s)\left(t-s^{-1}\right)$ where

$$
\operatorname{det} M=\Delta_{K}(s t) \Delta_{K}\left(s^{-1} t\right) \text {. }
$$

Here we remark that the top coefficient of $\Delta_{K, \rho^{\prime}}(t)$ remains the constant 1 by our assumption. Since $K$ is a nonfibered 2-bridge knot (in particular a nonfibered alternating knot), the Alexander polynomial $\Delta_{K}(t)$ is nonmonic (see [26, Theorem 1.2]). Hence the right hand side of (4.1) is also nonmonic, but this is a contradiction because $\Delta_{K, \rho^{\prime}}(t)$ is monic if and only if the numerator of $\Delta_{K, \rho^{\prime}}(t)$, which is $\operatorname{det} M$, is monic.

The above theorem can be strengthened further. Namely, for a nonfibered 2bridge knot in $S^{3}$, we can find a special curve component of the character variety which contains only finitely many monic characters:

Theorem 4.2. For a nonfibered 2-bridge knot $K$, there exists an irreducible curve component in $X^{\mathrm{nab}}(K)$ which contains only a finite number of monic characters.

Proof. As we saw in the proof of Theorem 4.1 the Riley polynomial $\phi(x, y)$ of $K$ has an irreducible factor $\phi_{1}(x, y) \in \mathbb{C}[x, y]$ such that $\phi_{1}\left(s_{0}+s_{0}^{-1}, 2\right)=0$ for some $s_{0} \in \mathbb{C}^{*}$. Let $X_{1}$ be the curve given by $\phi_{1}(x, y)=0$. In particular, $X_{1}$ contains a character of a reducible nonabelian representation of $K$. By Remark 3.1 (iii) we may assume that for $\chi=\chi(x, y) \in X_{1}$,

$$
\Delta_{K, \chi}(t)=\psi_{4 g-2}(x, y) t^{4 g-2}+\psi_{4 g-3}(x, y) t^{4 g-3}+\cdots+\psi_{1}(x, y) t+\psi_{0}(x, y) .
$$

Then $\Delta_{K, \chi}(t)$ is monic if $\psi_{4 g-2}(x, y)=1$ or there exists $j \geq 2 g-1$ such that $\psi_{i}(x, y)=0$ for $j<i \leq 4 g-2$ and $\psi_{j}(x, y)=1$. Therefore the set of monic characters in the irreducible curve component $X_{1}$ is contained in $\left\{(x, y) \in \mathbb{C}^{2} \mid\right.$ $\phi_{1}(x, y)=0$ and $\left.\psi_{4 g-2}(x, y)=1\right\} \cup\left\{(x, y) \in \mathbb{C}^{2} \mid \phi_{1}(x, y)=0\right.$ and $\psi_{4 g-2}(x, y)=$ $0\}$. By our choice of $\phi_{1}, \psi_{4 g-2}(x, y)$ is not identically one on $X_{1}$ as was shown in the proof of Theorem 4.1. Also since $K$ is alternating, $\operatorname{deg}\left(\Delta_{K}(t)\right)=2 g$ (see [4, 25]). Therefore by our choice of $\phi_{1}$ and Remark 3.1 (iv), $\psi_{4 g-2}(x, y)$ is not identically 0 on $X_{1}$. Now we can conclude that $\phi_{1}(x, y)$ and $\psi_{4 g-2}(x, y)-1$ have no common divisors, and the affine plane curves defined by them have at most finitely many intersection points (Bézout's Theorem). Similarly, $\phi_{1}(x, y)=0$ and $\psi_{4 g-2}(x, y)=0$ have at most finitely many intersection points. Therefore the number of monic characters on $X_{1}$ is finite.

Note that in the above proof we do not need non-fiberedness of a 2-bridge knot to show that $\psi_{4 g-2}(x, y)$ is not identically 0 . Therefore $\phi_{1}(x, y)=0$ and $\psi_{4 g-2}(x, y)=$ 0 have at most finitely many intersection points for any 2-bridge knot, and we obtain the following theorem:

Theorem 4.3. For a 2-bridge knot $K$ of genus $g$, there exists an irreducible curve component $X_{1}$ in $X^{\mathrm{nab}}(K)$ such that $\operatorname{deg}\left(\Delta_{K, \chi}(t)\right)=4 g-2$ for all but finitely many $\chi \in X_{1}$. 
Remark 4.4. (i) It is known that for any positive integer $n$ there is a hyperbolic 2-bridge knot $K$ such that $X^{\text {irr }}(K)$ has at least $n$ irreducible components (see [29, Corollary 7.3]).

(ii) For a knot $K$ of genus $g$, in 8 . Dunfield, Friedl and Jackson show that the set of monic characters in $X(K)$ is Zariski closed and $\{\chi \in X(K) \mid$ $\left.\operatorname{deg}\left(\Delta_{K, \chi}(t)\right)=4 g-2\right\}$ is Zariski open.

Assuming a suitable condition for the 2-bridge knot $K(\alpha, \beta)$, we obtain the following finiteness result.

Theorem 4.5. Let $K=K(\alpha, \beta)$ be a nonfibered 2 -bridge knot and $c \in \mathbb{Z}$ the leading coefficient of $\Delta_{K}(t)$. Let $p$ be an odd prime divisor of $\alpha$. Suppose $c \not \equiv 0$ and $c^{2} \not \equiv$ $\pm 1 \bmod p$. Then the number of monic characters in $X^{\mathrm{nab}}(K)$ is finite.

Proof. By Lemmas 4.6 and 4.8 below, we see that the coefficient of the highest degree term of the twisted Alexander polynomials is not identically one on each irreducible component of $X^{\mathrm{nab}}(K)$. Therefore, by the arguments in the proof of Theorem 4.2 the number of monic characters is finite for each irreducible component of $X^{\mathrm{nab}}(K)$.

A representation $\rho: G(K) \rightarrow S L(2, \mathbb{C})$ is called metabelian if the image of the commutator subgroup $[G(K), G(K)]$ is an abelian subgroup in $S L(2, \mathbb{C})$. It should be noted that reducible representations are metabelian by [5, Lemma 1.2.1] (see also [27, Section 2]).

Lemma 4.6. Any irreducible component of $X^{\mathrm{nab}}(K)$ of a 2-bridge knot $K$ contains a character of an irreducible metabelian representation.

Proof. As a polynomial in $\mathbb{Z}\left[s^{ \pm 1}\right][y]$, the coefficient of the highest degree term of the Riley polynomial $\phi(s, y)$ is \pm 1 (see [30, p.197]). Therefore $\phi(s, y)$ has the irreducible factorization

$$
\phi(s, y)= \pm \phi_{1}(s, y) \phi_{2}(s, y) \cdots \phi_{m}(s, y)
$$

over $\mathbb{Z}\left[s^{ \pm 1}\right][y]$ such that each $\phi_{k}(s, y)$ is a monic polynomial in $\mathbb{Z}\left[s^{ \pm 1}\right][y]$. By Lemma 3 and Proposition 1 in 30 , it follows that $\phi_{k}(s, y)=\phi_{k}\left(s^{-1}, y\right)$ for any $k$. We then put $x=s+s^{-1}$ and regard each $\phi_{k}$ as an element of $\mathbb{Z}[x, y]$. It might be a reducible polynomial over $\mathbb{C}[x, y]$, but we see that $\phi_{k}(x, y)$ can be written as the product of monic polynomials in $\mathbb{C}[x][y]$, i.e. $\phi_{k}(x, y)=\phi_{k 1}(x, y) \phi_{k 2}(x, y) \cdots \phi_{k n_{k}}(x, y)$, where each $\phi_{k \ell}(x, y)$ is an irreducible monic polynomial in $\mathbb{C}[x][y]$. Therefore, every irreducible component $\phi_{k \ell}(x, y)=0$ contains a character of the representation corresponding to $s=i$ where $i=\sqrt{-1}$. In other words, $\phi_{k \ell}(i+1 / i, y)=0$ has a solution for $y$ for any $k, \ell$. Since the character of this representation is 0 , the representation is irreducible metabelian (see [27, Section 4]).

Let $K$ be a 2-bridge knot $K(\alpha, \beta)$ and fix a Wirtinger presentation $G(K)=$ $\langle a, b \mid w a=b w\rangle$. If $p=2 n+1$ is a prime divisor of $\alpha$, then as in [15, Section 2] one can define a representation $\xi: G(K) \rightarrow G L(2, \mathbb{C})$ by

$$
\xi(a)=\left(\begin{array}{cc}
-1 & 1 \\
0 & 1
\end{array}\right), \quad \xi(b)=\left(\begin{array}{cc}
-1 & 0 \\
\omega & 1
\end{array}\right)
$$

where $\omega \in \mathbb{C}$ is an algebraic integer whose minimal polynomial over $\mathbb{Z}$ is $z^{n}+$ $c_{n-1}^{(n)} z^{n-1}+\cdots+c_{1}^{(n)} z+p$ for some $c_{j}^{(n)} \in \mathbb{Z}, 1 \leq j \leq n-1$. 
Lemma 4.7. Let $K=K(\alpha, \beta)$ be a 2-bridge knot of genus $g$ and $c \in \mathbb{Z}$ the leading coefficient of $\Delta_{K}(t)$. Let $p=2 n+1$ be a prime divisor of $\alpha$. Suppose $c \neq \equiv 0 \bmod p$. Then the degree of $\Delta_{K, \xi}(t)$ is $4 g-2$.

Proof. We calculate $\Delta_{K, \xi}(t)$ according to [15, Section 7]. Put $r=w a w^{-1} b^{-1}$. As was shown in [15, p.17] we obtain

$$
\Phi\left(\frac{\partial r}{\partial a}\right)=\left(\begin{array}{cc}
\Delta_{K}(-t)+\omega \mu_{11} & \mu_{12} \\
\omega \mu_{21} & \Delta_{K}(t)+\omega \mu_{11}
\end{array}\right)
$$

where $\mu_{i j} \in \mathbb{Z}[\omega]\left[t^{ \pm 1}\right]$. Then $\Delta_{K, \xi}(t)=\operatorname{det} \Phi\left(\frac{\partial r}{\partial a}\right) /\left(1-t^{2}\right)$. Since $K$ is a 2-bridge knot, it is alternating, and therefore $\operatorname{deg}\left(\Delta_{K}(t)\right)=2 g$ (see [4, 25]). Therefore we can write $\Delta_{K}(t)=c t^{l}+b_{l-1} t^{l-1}+\cdots+b_{m+1} t^{m+1}+c t^{m}$ where $l-m=2 g$ and $b_{j} \in \mathbb{Z}(m+1 \leq j \leq l-1)$. Then one can see that each $\mu_{i j}$ is written as $a_{l}^{i j} t^{l}+a_{l-1}^{i j} t^{l-1}+\cdots+a_{m+1}^{i j} t^{m+1}+a_{m}^{i j} t^{m}$ for some $a_{k}^{i j} \in \mathbb{Z}[\omega]$ (see [15, Section 7]).

Therefore we obtain that $\Delta_{K, \xi}(t)=\psi_{2 l-2} t^{2 l-2}+\psi_{2 l-1} t^{2 l-1}+\cdots+\psi_{2 m+1} t^{2 m+1}+$ $\psi_{2 m} t^{2 m}$ for some $\psi_{k} \in \mathbb{Z}[\omega], 2 m \leq k \leq 2 l-2$. We will show that $\psi_{2 l-2} \neq 0$ and $\psi_{2 m} \neq 0$, which will complete the proof.

Since the minimal polynomial of $\omega$ over $\mathbb{Z}$ is $z^{n}+c_{n-1}^{(n)} z^{n-1}+\cdots+c_{1}^{(n)} z+p$,

$$
\psi_{2 l-2}=c^{2}+p c_{0}+c_{1} \omega+\cdots+c_{n-1} \omega^{n-1}
$$

for some $c_{j} \in \mathbb{Z}$. If $c_{j} \neq 0$ for some $j(1 \leq j \leq n-1)$, then $\psi_{2 l-2} \neq 0$. Otherwise $\psi_{2 l-2}=c^{2}+p c$, and since $c \not \equiv 0 \bmod p, \psi_{2 l-2}$ is nonzero. Similarly, one can prove that $\psi_{2 m} \neq 0$.

Lemma 4.8. Let $K=K(\alpha, \beta)$ be a 2-bridge knot and $c \in \mathbb{Z}$ the leading coeffcient of $\Delta_{K}(t)$. Let $p=2 n+1$ be a prime divisor of $\alpha$. Suppose $c \not \equiv 0$ and $c^{2} \not \equiv$ $\pm 1 \bmod p$. Then for any irreducible metabelian representation $\rho, \Delta_{K, \rho}(t)$ is nonmonic.

Note that $c \neq \pm 1$ by the assumption, and therefore the knot $K$, which is alternating, is not fibered.

Proof. Fix a Wirtinger presentation $G(K)=\langle a, b \mid w a=b w\rangle$. Since $\rho$ is irreducible metabelian, the character of $\rho$ is 0 (see [27, Section 4]). Therefore we may assume that

$$
\rho(a)=\left(\begin{array}{cc}
i & 1 \\
0 & -i
\end{array}\right), \quad \rho(b)=\left(\begin{array}{cc}
i & 0 \\
2-y_{0} & -i
\end{array}\right)
$$

for some $y_{0} \in \mathbb{C}$. Let $\xi: G(K) \rightarrow G L(2, \mathbb{C})$ be the representation defined in the paragraph preceding Lemma 4.7. Then we can easily check that $\xi$ is conjugate to $i \cdot \rho$ and $\Delta_{K, \rho}(t)=\Delta_{K, \xi}(-i t)$ holds. Since the highest exponent of $\Delta_{K, \xi}(t)$ is $2 l-2$ as we calculated in the proof of Lemma 4.7, this implies that if the leading coefficient of $\Delta_{K, \xi}(t)$ is not \pm 1 , then $\Delta_{K, \rho}(t)$ is nonmonic. is

By the assumption, as in the proof of Lemma 4.7 the leading coefficient of $\Delta_{K, \xi}(t)$

$$
\psi_{2 l-2}=c^{2}+p c_{0}+c_{1} \omega+\cdots+c_{n-1} \omega^{n-1}
$$

for some $c_{j} \in \mathbb{Z}$. If $c_{j} \neq 0$ for some $j(1 \leq j \leq n-1)$, then $\psi_{2 l-2} \neq \pm 1$. If $c_{j}=0$ for any $1 \leq j \leq n-1$, then $\psi_{2 l-2}=c^{2}+p c_{0}$. Since $c^{2}+p c_{0} \equiv c^{2} \not \equiv \pm 1 \bmod p$, we have $c^{2}+p c_{0} \neq \pm 1$. Therefore $\psi_{2 l-2} \neq \pm 1$.

Using the above lemmas, we can obtain the following theorem which extends Theorem 4.3 under a certain condition. 
Theorem 4.9. Let $K=K(\alpha, \beta)$ be a 2-bridge knot of genus $g$ and $c \in \mathbb{Z}$ the leading coefficient of $\Delta_{K}(t)$. Let $p$ be an odd prime divisor of $\alpha$. Suppose $c \not \equiv 0 \bmod p$. Then $\operatorname{deg}\left(\Delta_{K, \chi}(t)\right)=4 g-2$ for all but finitely many $\chi \in X^{\mathrm{nab}}(K)$.

Proof. By Lemma 4.6, any irreducible component of $X^{\text {nab }}$ contains a metabelian representation, say $\rho$. From Lemma 4.7 and the proof of Lemma 4.8, one can see that $\operatorname{deg}\left(\Delta_{K, \rho}(t)\right)=4 g-2$. Following the notations in the proof of Theorem 4.2 , for $\chi=\chi(x, y) \in X^{\text {nab }}$ let us write

$$
\Delta_{K, \chi}(t)=\psi_{4 g-2}(x, y) t^{4 g-2}+\psi_{4 g-3}(x, y) t^{4 g-3}+\cdots+\psi_{1}(x, y) t+\psi_{0}(x, y) .
$$

Then we have $\operatorname{deg}\left(\Delta_{K, \chi}(t)\right)<4 g-2$ exactly when $\psi_{4 g-2}(x, y)=0$ or $\psi_{0}(x, y)=0$. Since on each irreducible component of $X^{\text {nab }}$ there exists a (metabelian) character whose associated twisted Alexander polynomial is of degree $4 g-2, \psi_{4 g-2}(x, y)$ is not identically zero on every irreducible component $\phi_{k \ell}(x, y)=0$. Similarly, $\psi_{0}(x, y)$ is not identically zero on $\phi_{k \ell}(x, y)=0$, either. Therefore by Bézout's Theorem, $\phi(x, y)=0$ and $\psi_{4 g-2}(x, y)=0$ have finitely many intersection points, and so do $\phi(x, y)=0$ and $\psi_{0}(x, y)=0$.

Example 4.10. Let $K$ be the 2-bridge knot $K(7,3)=5_{2}$ with the Alexander polynomial $\Delta_{K}(t)=2 t^{2}-3 t+2$. This is one of twist knots and hence has genus one. By Theorem 4.5, the number of monic characters in $X^{\mathrm{nab}}(K)$ is finite. Moreover, in this example, we can directly find monic characters as follows. The knot group $G(K)$ has a presentation

$$
G(K)=\langle a, b \mid w a=b w\rangle, \quad w=\left[b, a^{-1}\right]^{2} .
$$

We then see that the polynomial $\phi(x, y)$ is given by an irreducible polynomial

$$
\phi(x, y)=1-4 x^{2}+2 x^{4}+\left(2-x^{2}-x^{4}\right) y-\left(1-2 x^{2}\right) y^{2}-y^{3} .
$$

The twisted Alexander polynomial in this case is

$$
\Delta_{K, \chi}(t)=\psi_{2}(x, y) t^{2}+\psi_{1}(x, y) t+\psi_{0}(x, y),
$$

where $\psi_{2}(x, y)=\psi_{0}(x, y)=2 x^{2}-x^{2} y+y^{2}$ and $\psi_{1}(x, y)=-2 x$. An easy calculation shows that $\left\{(x, y) \in \mathbb{C}^{2} \mid \phi(x, y)=0, \psi_{2}(x, y)=0\right.$ and $\left.\psi_{1}(x, y)=1\right\}$ is an empty set and

$$
\left\{(x, y) \in \mathbb{C}^{2} \mid \phi(x, y)=0 \text { and } \psi_{2}(x, y)=1\right\}=\left( \pm \frac{1}{\sqrt{2}}, \frac{1}{2}\right),
$$

namely there are only two intersection points (see [24, Example 5.4]).

Next by Theorem [4.9, $\operatorname{deg}\left(\Delta_{K, \chi}(t)\right)=4 \cdot 1-2=2$ for all but finitely many $\chi \in X^{\mathrm{nab}}(K)$. In fact, the similar calculation as above shows that

$$
\left\{(x, y) \in \mathbb{C}^{2} \mid \phi(x, y)=0 \text { and } \psi_{2}(x, y)=0\right\}=\left( \pm \frac{i}{\sqrt{6}},-\frac{2}{3}\right) .
$$

Therefore except for these two points the twisted Alexander polynomial $\Delta_{K, \chi}(t)$ is of degree 2 .

\section{A BOUND FOR 2-BRIDGE KNOTS}

In the previous section, we discussed finiteness of the number of monic characters for general 2-bridge knots. However, Theorem 4.2 or Theorem 4.5 says nothing about upper bounds of the number of monic characters. In this section, we give a bound for a family of certain 2-bridge knots. 
5.1. A family of 2-bridge knots. Let $K=J(k, l)$ be a knot as in Figure 1 where $k$ and $l$ are integers corresponding to the number of half twists in the labeled boxes. Positive numbers correspond to right-handed twists and negative numbers

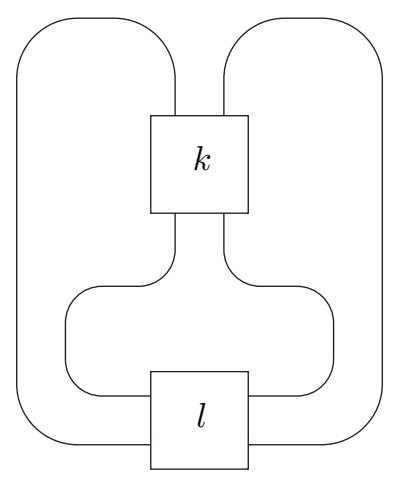

Figure 1. The knot $J(k, l)$

correspond to left-handed twists respectively. The knot $J(k, l)$ is ambient isotopic to the 2 -bridge knot $K(\alpha, \beta)$ such that $\frac{\beta}{\alpha}$ is equal to $\frac{l}{1-k l}$ in $\mathbb{Q} / \mathbb{Z}$. We remark that $J(k, l)$ is a knot if and only if $k l$ is even (otherwise it is a 2-component link). It has an obvious symmetry with switching $k$ and $l$. Moreover $J(-k,-l)$ is the mirror image of $J(k, l)$. Thus, in the following, we only consider the case where $K=J(k, 2 q)$ for $k>0$ and $q \in \mathbb{Z}$. It is easy to see that $K=J(2,2 q)$ is a twist knot and this family contains the trefoil knot $J(2,2)$ and the figure eight knot $J(2,-2)$.

The knot group $G(K)$ for $K=J(k, 2 q)$ has a presentation

$$
G(K)=\left\langle a, b \mid\left(w_{m}\right)^{q} a=b\left(w_{m}\right)^{q}\right\rangle
$$

where

$$
w_{m}= \begin{cases}\left(b a^{-1}\right)^{m}\left(b^{-1} a\right)^{m}, & k=2 m \\ \left(b a^{-1}\right)^{m} b a\left(b^{-1} a\right)^{m}, & k=2 m+1\end{cases}
$$

see [16, Proposition 1]. Let $\rho: G(K) \rightarrow S L(2, \mathbb{C})$ be a nonabelian representation defined by the correspondence

$$
\rho(a)=\left(\begin{array}{cc}
s & 1 \\
0 & s^{-1}
\end{array}\right) \quad \text { and } \quad \rho(b)=\left(\begin{array}{cc}
s & 0 \\
-y & s^{-1}
\end{array}\right),
$$

where $s \neq 0, y \in \mathbb{C}$. For simplicity of calculations, we have slightly modified the parametrization of $\hat{R}^{\text {nab }}(K)$. We also denote $\rho(a), \rho(b)$ and $\rho\left(w_{m}\right)$ by $A, B$ and $W_{m}$ respectively.

The next theorem is a generalization of our previous result (see 13, Theorem 1.2] and [24, Theorem 5.3]) for the twist knot $K=J(2,2 q)$.

Theorem 5.1. Let $K=J(k, 2 q)$ be a nonfibered knot, where $k>0, q \in \mathbb{Z}$ and $k \neq 2 q$. Then the number of monic characters is bounded above by

$$
2(k+1)^{2} q^{2}-(k+1)(k+4)|q|
$$

if $k$ is even or

if $k$ is odd.

$$
(k+1)(k-1)|q|
$$


Proof. It is known that $J(k, 2 q)$ is fibered only for the trivial knot $J(k, 0)$, the trefoil knot $J(2,2)$, the figure eight knot $J(2,-2)$, the knots $J(1,2 q)$ for any $q$ (it is the torus $(2,2 q-1)$-knot) and $J(3,2 q)$ for $q>0$. Therefore the assertion immediately follows from the fact that the character variety $X^{\text {nab }}(J(k, 2 q))$ is irreducible when $k \neq 2 q$ (see [23, Theorem 1.2]), and Propositions 5.2 and 5.3. That is, the upper bound is given by twice the product of the degrees of two polynomials $\phi(x, y)$ and $\psi_{4 g-2}(x, y)$. In fact, $\left\{(x, y) \in \mathbb{C}^{2} \mid \phi(x, y)=0\right.$ and $\left.\psi_{4 g-2}(x, y)=1\right\}$ consists of at most $(k+1)^{2} q^{2}-(k+1)(k+4)|q| / 2$ points if $k$ is even or $(k+1)(k-1)|q| / 2$ points if $k$ is odd (Bézout's Theorem). Similarly, $\left\{(x, y) \in \mathbb{C}^{2} \mid \phi(x, y)=0\right.$ and $\left.\psi_{4 g-2}(x, y)=0\right\}$ consists of at most the same number of points, because $\operatorname{deg}\left(\psi_{4 g-2}(x, y)-1\right)=$ $\operatorname{deg}\left(\psi_{4 g-2}(x, y)\right)$ clearly holds. This completes the proof of Theorem 5.1.

Since $x=s+s^{-1}$, the Riley polynomial $\phi(s, y) \in \mathbb{Z}\left[s^{ \pm 1}, y\right]$ and the polynomial $\phi(x, y)$ have the same degree. Here the exponent of the highest degree term of $\phi(s, y)$ is defined to be the degree of $\phi(s, y)$. Moreover, we recall that the top coefficient $\psi(s, y)$ is also a polynomial in variables $x=s+s^{-1}$ and $y$. Therefore $\psi(s, y)$ and $\psi(x, y)$ have the same degree. In the following, we consider $\phi$ and $\psi$ as polynomials in $s$ and $y$ rather than in $x$ and $y$. The next two propositions will be proved in Subsections 5.2 and 5.3 respectively.

Proposition 5.2. Let $\phi_{k, q}(s, y)$ be the Riley polynomial of $K=J(k, 2 q)$, where $k>0$ and $q \in \mathbb{Z}$. Then its degree is given by

$$
\operatorname{deg} \phi_{k, q}(s, y)= \begin{cases}(k+1) q-1, & q>0, \quad k \neq 1 \\ (k+1)|q|, & q<0,\end{cases}
$$

and $\operatorname{deg} \phi_{1, q}(s, y)=2 q-2(q>0)$.

Proposition 5.3. Let $\psi_{k, q}(s, y)$ be the coefficient of the highest degree term of $\Delta_{K, \rho}(t)$ for $K=J(k, 2 q)$, where $k>0$ and $q \in \mathbb{Z}$. Then its degree is given by

$$
\operatorname{deg} \psi_{k, q}(s, y)= \begin{cases}(k+1)|q|-\frac{k+4}{2}, & k \text { is even; } \\ \frac{k-3}{2}, & k \neq 1 \text { is odd, } q>0 ; \\ \frac{k-1}{2}, & k \text { is odd, } q<0,\end{cases}
$$

and $\operatorname{deg} \psi_{1, q}(s, y)=0$ for $q>0$.

Example 5.4. Let $K=J(4,4)=7_{4}$. As we have seen in Example 2.2, $\phi_{4,2}(s, y)$ has the factorization $\phi_{4,2}=\phi_{1} \phi_{2}$. Then each factor defines an irreducible curve (namely $X^{\text {nab }}(K)$ has two components, see [23, Theorem 1.2]). So Theorem 5.1 does not work. Moreover Theorem 4.5 cannot be applied to $K=7_{4}$, because $c=4$ and $p=3,5$ for the $\operatorname{knot} K$, i.e. $c^{2} \equiv 1 \bmod p$. However in this example, the existence of a parabolic representation on $\phi_{2}(s, y)=0$ (it is given by $\phi_{2}(1, y)=0$ ) ensures finiteness of the number of monic characters on the whole $X^{\text {nab }}(K)$. In fact, we can show that the twisted Alexander polynomial associated with the parabolic representation is nonmonic.

Remark 5.5. In the case of $k=2 q$, the character variety $X(J(k, k))$ is reducible and has two components (see [23). This phenomenon follows from the fact that the canonical component $X_{0}(J(k, k))$ (see Section 6$)$ is fixed by the involution induced by turning the 4-plat presentation upside down, while other components are not (see [28]). 
5.2. Proof of Proposition 5.2. We first consider the case where $K=J(k, 2 q)$ for $k=2 m(m>0)$ and $q>0$.

Lemma 5.6. For $W_{m}=\left(B A^{-1}\right)^{m}\left(B^{-1} A\right)^{m}=\left(w_{m}^{i j}\right)$, we have

$$
\operatorname{deg} w_{m}^{11}=\operatorname{deg} w_{m}^{21}=2 m+1 \text { and } \operatorname{deg} w_{m}^{12}=\operatorname{deg} w_{m}^{22}=2 m .
$$

In particular, $\operatorname{deg}\left(\operatorname{tr} W_{m}\right)=2 m+1$.

Proof. We show them by the inductive argument on $m$. When $m=1$, we can easily obtain

$$
\begin{aligned}
W_{1} & =B A^{-1} B^{-1} A=\left(\begin{array}{cc}
1 & -s \\
-s^{-1} y & y+1
\end{array}\right)\left(\begin{array}{cc}
1 & s^{-1} \\
s y & y+1
\end{array}\right) \\
& =\left(\begin{array}{cc}
1-s^{2} y & s^{-1}-s(y+1) \\
-s^{-1} y+s y(y+1) & -s^{-2} y+(y+1)^{2}
\end{array}\right) .
\end{aligned}
$$

Hence, $\operatorname{deg} w_{1}^{11}=\operatorname{deg} w_{1}^{21}=3$ and $\operatorname{deg} w_{1}^{12}=\operatorname{deg} w_{1}^{22}=2$ as desired. In particular, we note that the highest degree term in each entry $w_{1}^{i j}$ does not contain the negative power of $s$.

Next for the general case, we have

$$
\begin{aligned}
W_{m+1} & =\left(B A^{-1}\right)^{m+1}\left(B^{-1} A\right)^{m+1}=\left(B A^{-1}\right) W_{m}\left(B^{-1} A\right) \\
& =\left(\begin{array}{cc}
1 & -s \\
-s^{-1} y & y+1
\end{array}\right)\left(\begin{array}{ll}
w_{m}^{11} & w_{m}^{12} \\
w_{m}^{21} & w_{m}^{22}
\end{array}\right)\left(\begin{array}{cc}
1 & s^{-1} \\
s y & y+1
\end{array}\right)=\left(\begin{array}{ll}
w_{m+1}^{11} & w_{m+1}^{12} \\
w_{m+1}^{21} & w_{m+1}^{22}
\end{array}\right),
\end{aligned}
$$

where

$$
\begin{aligned}
& w_{m+1}^{11}=w_{m}^{11}-s w_{m}^{21}+s y w_{m}^{12}-s^{2} y w_{m}^{22}, \\
& w_{m+1}^{12}=s^{-1} w_{m}^{11}-w_{m}^{21}+(y+1) w_{m}^{12}-s(y+1) w_{m}^{22}, \\
& w_{m+1}^{21}=-s^{-1} y w_{m}^{11}+(y+1) w_{m}^{21}-y^{2} w_{m}^{12}+s y(y+1) w_{m}^{22}, \\
& w_{m+1}^{22}=-s^{-2} y w_{m}^{11}+s^{-1}(y+1) w_{m}^{21}-s^{-1} y(y+1) w_{m}^{12}+(y+1)^{2} w_{m}^{22} .
\end{aligned}
$$

Using the assumption of the induction and checking the degree of each component carefully, we can conclude that $\operatorname{deg} w_{m+1}^{11}=\operatorname{deg} w_{m+1}^{21}=2 m+3$ and $\operatorname{deg} w_{m+1}^{12}=$ $\operatorname{deg} w_{m+1}^{22}=2 m+2$. In fact, $s^{2} y w_{m}^{22}$ and $s y(y+1) w_{m}^{22}$ attain the degree $2 m+3$, and $s(y+1) w_{m}^{22}$ and $(y+1)^{2} w_{m}^{22}$ attain the degree $2 m+2$. In particular, $s^{2} y w_{m}^{22}$, which attains the degree of $\operatorname{tr} W_{m+1}$, never contains the negative power of $s$.

Lemma 5.7. For $K=J(2 m, 2), \operatorname{deg} \phi_{2 m, 1}(s, y)=2 m$.

Proof. Using calculations in Lemma 5.6, when $m=1$, it follows that

$$
\phi_{2,1}(s, y)=w_{1}^{11}+\left(s^{-1}-s\right) w_{1}^{12}=s^{2}+s^{-2}-y-1 .
$$

Hence we find $\operatorname{deg} \phi_{2,1}(s, y)=2$. For the general case,

$$
\begin{aligned}
\phi_{2(m+1), 1} & =w_{m+1}^{11}+\left(s^{-1}-s\right) w_{m+1}^{12} \\
& =-s w_{m}^{12}+s^{2} w_{m}^{22}+s^{-1}\left(s^{-1} w_{m}^{11}-w_{m}^{21}+(y+1) w_{m}^{12}-s(y+1) w_{m}^{22}\right),
\end{aligned}
$$

so that we have $\operatorname{deg} \phi_{2(m+1), 1}(s, y)=2 m+2=2(m+1)$. In fact, $s^{2} w_{m}^{22}$ attains the degree $2 m+2$. 
We see from [16, Section 3] that there exists a recursive relation

$$
\phi_{k, q}=\left(\operatorname{tr} W_{m}\right) \phi_{k, q-1}-\phi_{k, q-2}
$$

for the Riley polynomial $\phi_{k, q}(s, y)$ of $K=J(k, 2 q)$. Thus we obtain

$$
\operatorname{deg} \phi_{k, q}=\operatorname{deg} \phi_{k, q-1}+\operatorname{deg}\left(\operatorname{tr} W_{m}\right) \text {. }
$$

Therefore we see from Lemmas 5.6 and 5.7 that the degree of $\phi_{2 m, q}(s, y)$ is given by

$$
\begin{aligned}
\operatorname{deg} \phi_{2 m, q} & =\operatorname{deg} \phi_{2 m, 1}+(2 m+1)(q-1) \\
& =(2 m+1) q-1 .
\end{aligned}
$$

Next we consider the case where $K=J(k, 2 q)$ for $k=2 m+1(m \geq 0)$ and $q>0$. The following two lemmas are shown by the same argument as in Lemmas 5.6 and 5.7 .

Lemma 5.8. For $W_{m}=\left(B A^{-1}\right)^{m} B A\left(B^{-1} A\right)^{m}=\left(w_{m}^{i j}\right)$, we have

$$
\operatorname{deg} w_{m}^{11}=\operatorname{deg} w_{m}^{21}=2 m+2 \text { and } \operatorname{deg} w_{m}^{12}=\operatorname{deg} w_{m}^{22}=2 m+1 .
$$

In particular, $\operatorname{deg}\left(\operatorname{tr} W_{m}\right)=2 m+2$.

Lemma 5.9. For $K=J(2 m+1,2), \operatorname{deg} \phi_{1,1}(s, y)=0$ and $\operatorname{deg} \phi_{2 m+1,1}(s, y)=$ $2 m+1(m>0)$.

From Lemmas 5.8 and [5.9, we have $\operatorname{deg} \phi_{1, q}=2 q-2$ and

$$
\begin{aligned}
\operatorname{deg} \phi_{2 m+1, q} & =\operatorname{deg} \phi_{2 m+1,1}+(2 m+2)(q-1) \\
& =(2 m+2) q-1 .
\end{aligned}
$$

In the case of $q<0$, we can show the following lemma. The proof is almost the same as Lemmas 5.6, 5.7, 5.8 and 5.9,

Lemma 5.10. $\operatorname{deg} \phi_{2 m,-1}(s, y)=2 m+1$ and $\operatorname{deg} \phi_{2 m+1,-1}(s, y)=2 m+2$.

Since $\operatorname{deg}\left(\operatorname{tr} W_{m}^{-1}\right)=\operatorname{deg}\left(\operatorname{tr} W_{m}\right)$ holds, we can conclude that

$$
\begin{aligned}
\operatorname{deg} \phi_{k, q}(s, y) & =\operatorname{deg} \phi_{k,-1}(s, y)+(k+1)(|q|-1) \\
& =(k+1)|q| .
\end{aligned}
$$

This completes the proof of Proposition 5.2

5.3. Proof of Proposition 5.3. Putting $r=w_{m}^{q} a w_{m}^{-q} b^{-1}$, we have

$$
\begin{aligned}
\frac{\partial r}{\partial a} & =\frac{\partial w_{m}^{q}}{\partial a}+w_{m}^{q}\left(1+a \frac{\partial w_{m}^{-q}}{\partial a}\right) \\
& =\left(1+w_{m}+\cdots+w_{m}^{q-1}\right) \frac{\partial w_{m}}{\partial a}+w_{m}^{q}\left(1+a\left(1+w_{m}^{-1}+\cdots+w_{m}^{-q+1}\right) \frac{\partial w_{m}^{-1}}{\partial a}\right) \\
& =w_{m}^{q}\left(w_{m}^{-q}+\cdots+w_{m}^{-1}\right) \frac{\partial w_{m}}{\partial a}+w_{m}^{q}\left(1-a\left(w_{m}^{-1}+\cdots+w_{m}^{-q}\right) \frac{\partial w_{m}}{\partial a}\right) \\
& =w_{m}^{q}\left(1+(1-a)\left(w_{m}^{-1}+\cdots+w_{m}^{-q}\right) \frac{\partial w_{m}}{\partial a}\right),
\end{aligned}
$$

where

$$
\frac{\partial w_{m}}{\partial a}=-\left(b a^{-1}+\cdots+\left(b a^{-1}\right)^{m}\right)+\left(b a^{-1}\right)^{m}\left(1+b^{-1} a+\cdots+\left(b^{-1} a\right)^{m-1}\right) b^{-1}
$$


if $k=2 m$ or

$$
\frac{\partial w_{m}}{\partial a}=-\left(b a^{-1}+\cdots+\left(b a^{-1}\right)^{m}\right)+\left(b a^{-1}\right)^{m} b\left(1+a b^{-1}+\cdots+\left(a b^{-1}\right)^{m}\right)
$$

if $k=2 m+1$.

We consider the coefficient of the highest degree term of the twisted Alexander polynomial $\Delta_{K, \rho}(t)$ for $K=J(2 m, 2 q)$, where $m>0$ and $q>0$. The numerator of $\Delta_{K, \rho}(t)$ is given by

$$
\operatorname{det} M_{2}=\left|I+(I-t A)\left(W_{m}^{-1}+W_{m}^{-2}+\cdots+W_{m}^{-q}\right) V\right|,
$$

where

$$
V=-\left(B A^{-1}+\cdots+\left(B A^{-1}\right)^{m}\right)+\left(B A^{-1}\right)^{m}\left(I+B^{-1} A+\cdots+\left(B^{-1} A\right)^{m-1}\right) t^{-1} B^{-1} .
$$

Since the coefficient of $t^{2}$ in $\operatorname{det} M_{2}$ (i.e. the highest degree term of $\Delta_{K, \rho}(t)$ ) is

$$
\left|-A\left(W_{m}^{-1}+\cdots+W_{m}^{-q}\right)\left(B A^{-1}+\cdots+\left(B A^{-1}\right)^{m}\right)\right|,
$$

we would like to compute its degree. To this end, we first show the following.

Lemma 5.11. $\operatorname{deg}\left|I+B A^{-1}+\cdots+\left(B A^{-1}\right)^{m-1}\right|=m-1$.

Proof. When $m=1, \operatorname{deg}|I|=\operatorname{deg} 1=0$. Next we assume

$$
\operatorname{deg}\left|I+B A^{-1}+\cdots+\left(B A^{-1}\right)^{m-1}\right|=m-1 .
$$

A direct calculation shows that

$$
\begin{aligned}
\left|I+B A^{-1}+\cdots+\left(B A^{-1}\right)^{m}\right|= & \left|B A^{-1}+\cdots+\left(B A^{-1}\right)^{m}\right| \\
& +\operatorname{tr}\left(B A^{-1}+\cdots+\left(B A^{-1}\right)^{m}\right)+1 \\
= & \left|I+B A^{-1}+\cdots+\left(B A^{-1}\right)^{m-1}\right| \\
& +\operatorname{tr}\left(B A^{-1}+\cdots+\left(B A^{-1}\right)^{m}\right)+1,
\end{aligned}
$$

where $B A^{-1} \in S L(2, \mathbb{C})$ and the degree of the first term is $m-1$ by the assumption of the induction. Further we see from the following claim that the degree of the second term is $m$. Therefore we obtain

$$
\operatorname{deg}\left|I+B A^{-1}+\cdots+\left(B A^{-1}\right)^{m}\right|=m
$$

This completes the proof of Lemma 5.11 .

Claim. $\operatorname{deg}\left(\operatorname{tr}\left(B A^{-1}\right)^{m}\right)=m$.

Proof. We set $\left(B A^{-1}\right)^{m}=\left(v_{m}^{i j}\right)$. Since $B A^{-1}=\left(\begin{array}{cc}1 & -s \\ -s^{-1} y & y+1\end{array}\right)$ for $m=1$, $\operatorname{deg} v_{1}^{11}=\operatorname{deg} v_{1}^{21}=0$ and $\operatorname{deg} v_{1}^{12}=\operatorname{deg} v_{1}^{22}=1$ hold. Thus $\operatorname{deg}\left(\operatorname{tr} B A^{-1}\right)=1$. In particular, the term which attains the highest degree of $\operatorname{tr} B A^{-1}$ (it is $y$ in this case) does not contain the variable $s$.

Next we assume that $\operatorname{deg} v_{m}^{11}=\operatorname{deg} v_{m}^{21}=m-1$ and $\operatorname{deg} v_{m}^{12}=\operatorname{deg} v_{m}^{22}=m$.

$$
\begin{aligned}
\left(B A^{-1}\right)^{m+1} & =\left(\begin{array}{ll}
v_{m}^{11} & v_{m}^{12} \\
v_{m}^{21} & v_{m}^{22}
\end{array}\right)\left(\begin{array}{cc}
1 & -s \\
-s^{-1} y & y+1
\end{array}\right) \\
& =\left(\begin{array}{ll}
v_{m}^{11}-s^{-1} y v_{m}^{12} & -s v_{m}^{11}+(y+1) v_{m}^{12} \\
v_{m}^{21}-s^{-1} y v_{m}^{22} & -s v_{m}^{21}+(y+1) v_{m}^{22}
\end{array}\right)
\end{aligned}
$$

implies that $\operatorname{deg} v_{m+1}^{11}=\operatorname{deg} v_{m+1}^{21}=m$ and $\operatorname{deg} v_{m+1}^{12}=\operatorname{deg} v_{m+1}^{22}=m+1$. In particular, $y v_{m}^{22}$ in $(2,2)$-entry attains the desired degree and it does not contain $s$. Hence $\operatorname{deg}\left(\operatorname{tr}\left(B A^{-1}\right)^{m+1}\right)=m+1$. 
Lemma 5.12. $\operatorname{deg}\left|I+W_{m}^{-1}+\cdots+W_{m}^{-q+1}\right|=(2 m+1)(q-1)$.

Proof. When $q=1, \operatorname{deg}|I|=\operatorname{deg} 1=0$. For the general case, as in the proof of Lemma 5.11, we get

$$
\begin{aligned}
\left|I+W_{m}^{-1}+\cdots+W_{m}^{-q+1}\right|= & \left|W_{m}^{-1}+\cdots+W_{m}^{-q+1}\right| \\
& +\operatorname{tr}\left(W_{m}^{-1}+\cdots+W_{m}^{-q+1}\right)+1 \\
= & \left|I+W_{m}^{-1}+\cdots+W_{m}^{-q+2}\right| \\
& +\operatorname{tr}\left(W_{m}^{-1}+\cdots+W_{m}^{-q+1}\right)+1 .
\end{aligned}
$$

The degree of the first term is $(2 m+1)(q-2)$ by the assumption of the induction. On the other hand, we obtain

$$
\begin{aligned}
\operatorname{deg}\left(\operatorname{tr}\left(W_{m}^{-1}+\cdots+W_{m}^{-q+1}\right)\right) & =\operatorname{deg}\left(\operatorname{tr} W_{m}^{-q+1}\right) \\
& =(q-1) \operatorname{deg}\left(\operatorname{tr} W_{m}^{-1}\right) \\
& =(2 m+1)(q-1),
\end{aligned}
$$

because $\operatorname{deg}\left(\operatorname{tr} W_{m}^{-1}\right)=2 m+1$ by Lemma 5.6, and $\operatorname{deg}\left(\operatorname{tr} N^{q}\right)=q \operatorname{deg}(\operatorname{tr} N)$ holds for $N \in S L(2, \mathbb{C})$ in general. This completes the proof.

Since $A, B, W_{m} \in S L(2, \mathbb{C})$, Lemmas 5.11 and 5.12 show that

$$
\begin{aligned}
& \operatorname{deg} \mid-A\left(W_{m}^{-1}+\cdots+W_{m}^{-q}\right)\left(B A^{-1}+\cdots+\left(B A^{-1}\right)^{m} \mid\right. \\
= & \operatorname{deg}\left|I+W_{m}^{-1}+\cdots+W_{m}^{-q+1}\right|\left|I+B A^{-1}+\cdots+\left(B A^{-1}\right)^{m-1}\right| \\
= & \operatorname{deg}\left|I+W_{m}^{-1}+\cdots+W_{m}^{-q+1}\right|+\operatorname{deg}\left|I+B A^{-1}+\cdots+\left(B A^{-1}\right)^{m-1}\right| \\
= & (2 m+1) q-(m+2) .
\end{aligned}
$$

We finished the proof for $K=J(2 m, 2 q)$.

Next we consider the case where $K=J(k, 2 q)$ for $k=2 m+1(m \geq 0)$ and $q>0$. In this case, the numerator of $\Delta_{K, \rho}(t)$ is given by

$$
\operatorname{det} M_{2}=t^{4 q}\left|I+(I-t A)\left(t^{-2} W_{m}^{-1}+t^{-4} W_{m}^{-2}+\cdots+t^{-2 q} W_{m}^{-q}\right) V\right|
$$

where

$$
V=-\left(B A^{-1}+\cdots+\left(B A^{-1}\right)^{m}\right)+t\left(B A^{-1}\right)^{m} B\left(I+A B^{-1}+\cdots+\left(A B^{-1}\right)^{m}\right) .
$$

An easy calculation shows that the coefficient of the highest degree term in $\operatorname{det} M_{2}$ coincides with the top coefficient of

$$
\left|I-t^{2} A\left(t^{-2} W_{m}^{-1}+\cdots+t^{-2 q} W_{m}^{-q}\right)\left(B A^{-1}\right)^{m} B\left(I+A B^{-1}+\cdots+\left(A B^{-1}\right)^{m}\right)\right| .
$$

We first consider the case where $q=1$.

Lemma 5.13. The degree of

$$
\left|I-A W_{m}^{-1}\left(B A^{-1}\right)^{m} B\left(I+A B^{-1}+\cdots+\left(A B^{-1}\right)^{m}\right)\right|
$$

is $m-1$ for $m>0$ and 0 for $m=0$.

Proof. Since $W_{m}^{-1}=\left(B^{-1} A\right)^{-m} A^{-1} B^{-1}\left(B A^{-1}\right)^{-m}$, we have

$$
\begin{aligned}
& \left|I-A W_{m}^{-1}\left(B A^{-1}\right)^{m} B\left(I+A B^{-1}+\cdots+\left(A B^{-1}\right)^{m}\right)\right| \\
= & \left|I-\left(B A^{-1}\right)^{m}\left(I+A B^{-1}+\cdots+\left(A B^{-1}\right)^{m}\right)\right| \\
= & \left|I+B A^{-1}+\cdots+\left(B A^{-1}\right)^{m-1}\right| .
\end{aligned}
$$


We see from Lemma 5.11 that its degree is $m-1$. When $m=0, \operatorname{deg}\left|I-A W_{0}^{-1} B\right|=$ $\operatorname{deg}|O|=0$, where $O$ denotes the zero matrix.

For the general case, $t^{-2 q} W_{m}^{-q}$ is newly added under the assumption of the induction on $q$, but it never contributes the coefficient of the highest degree term. Because it has a negative power with the variable $t$. Namely, the degree of the top coefficient of $\Delta_{K, \rho}(t)$ is constant for the knots $K=J(2 m+1, q)$ when $m$ is fixed. Then it is equal to $m-1=\frac{k-3}{2}(k>1)$ or $0(k=1)$ by Lemma 5.13 ,

Finally, if we consider $\operatorname{det} M_{1}=\Phi\left(\frac{\partial r}{\partial b}\right)$ instead of $\operatorname{det} M_{2}=\Phi\left(\frac{\partial r}{\partial a}\right)$ for the case of $K=J(k, 2 q)$, where $k>0$ and $q<0$, then we can obtain the following lemma along the line of discussion above.

Lemma 5.14. The degree of the coefficient of the highest degree term is given by

$$
\operatorname{deg} \psi_{k, q}(s, y)= \begin{cases}(k+1)(-q)-\left(\frac{k}{2}+2\right), & k=2 m, q<0 ; \\ \frac{k-1}{2}, & k=2 m+1, q<0 .\end{cases}
$$

We omit the proof of Lemma 5.14 and this completes the proof of Proposition 5.3.

\section{Concluding Remarks}

As is well-known, each 2-bridge knot is a torus $(2,2 q-1)$-knot or a hyperbolic knot. Hence nonfibered 2-bridge knots are all hyperbolic knots. For a hyperbolic knot $K$ in $S^{3}$, it is well-known that there is, uniquely up to conjugation, a discrete faithful representation $\bar{\rho}_{0}: G(K) \rightarrow \operatorname{Isom}^{+}\left(\mathbb{H}^{3}\right) \cong P S L(2, \mathbb{C})$ such that $\mathbb{H}^{3} / G(K) \cong S^{3}-K$. Then, due to Thurston, $\bar{\rho}_{0}$ can be lifted to a discrete faithful representation $\rho_{0}: G(K) \rightarrow S L(2, \mathbb{C})$ (see [5, Proposition 3.1.1]). For the representation $\rho_{0}$, it is natural to raise the following conjecture.

Conjecture 6.1 (Dunfield-Friedl-Jackson [8]). The twisted Alexander polynomial $\Delta_{K, \rho_{0}}(t)$ detects all hyperbolic fibered knots.

Remark 6.2. They have shown in 8 that Conjecture 6.1 is true for all hyperbolic knots with at most thirteen crossings.

Now it is easy to see that the character variety $X(K)$ always contains a curve corresponding to abelian representations. If $K$ is a hyperbolic knot, by Thurston's Dehn surgery theorem, there is a so-called canonical component $X_{0}(K)$ in $X(K)$ which is a curve containing the character of a discrete faithful representation $\rho_{0}$ (see [6, Proposition 2]).

More recently, the work of Kronheimer-Mrowka (see also [1) establishes the next general fact.

Theorem 6.3. 20] Let $K$ be a nontrivial knot. Then $X^{\mathrm{nab}}(K)$ contains a curve for which all but finitely many of its elements are characters of irreducible representations.

In our point of view to the fibering problem, we conclude the present paper with the following conjecture.

Conjecture 6.4. For a nonfibered knot $K$, there exists a curve component $X_{1}(K)$ in $X^{\mathrm{nab}}(K)$ so that $\left\{\chi \in X_{1}(K) \mid \Delta_{K, \chi}(t)\right.$ is monic $\}$ is a finite set. 
Remark 6.5. If $K=J(k, l)$ is a nonfibered (hence hyperbolic) knot, the canonical component in $X^{\mathrm{nab}}(K)$ has a finite number of monic characters: if $k \neq l, X^{\mathrm{nab}}(K)$ is irreducible (hence it is the canonical component) by [23. Theorem 1.2] and it follows from Theorem 4.2. If $k=l$, by [23, Proposition 4.6] one can see that the canonical component has a reducible representation. Thus following the arguments in Theorems 4.1 and 4.2, we see that the canonical component has finitely many monic characters. Therefore one may further conjecture that for a nonfibered hyperbolic knot there are only finitely many monic characters in the canonical component. We do not know if this conjecture is true for 2-bridge knots.

Acknowledgements. The authors would like to thank Michel Boileau, Francis Bonahon, Teruaki Kitano, Fumikazu Nagasato and Robert Penner for helpful comments. The authors also thank Stefan Friedl for helpful comments and correcting the proof of Theorem 4.2. The first author was supported by the National Research Foundation of Korea(NRF) grant funded by the Korea government(MEST) (No. 20090068877 and 2009-0086441). The second author is supported in part by the Grantin-Aid for Scientific Research (No. 20740030), the Ministry of Education, Culture,

Sports, Science and Technology, Japan.

\section{REFERENCES}

1. M. Boileau, S. Boyer, A. W. Reid and S. Wang, Simon's conjecture for 2-bridge knots, arXiv:0903.2898.

2. G. Burde, Darstellungen von Knotengruppen, Math. Ann. 173 (1967), 24-33.

3. J. C. Cha, Fibred knots and twisted Alexander invariants, Trans. Amer. Math. Soc. 355 (2003), 4187-4200.

4. R. Crowell, Genus of alternating link types, Ann. of Math. (2) 69 (1959), 258-275.

5. M. Culler and P. B. Shalen, Varieties of group representations and splittings of 3-manifolds, Ann. of Math. 117 (1983), 109-146.

6. M. Culler and P. B. Shalen, Bounded, separating, incompressible surfaces in knot manifolds, Invent. Math. 75 (1984), no. 3, 537-545.

7. G. de Rham, Introduction aux polynomes d'un noeud, Enseignement Math. (2) 13 (1967), 187-194.

8. N. Dunfield, S. Friedl and N. Jackson, Twisted Alexander polynomials of hyperbolic knots, in preparation.

9. S. Friedl and T. Kim, The Thurston norm, fibered manifolds and twisted Alexander polynomials, Topology 45 (2006), 929-953.

10. S. Friedl and S. Vidussi, Twisted Alexander polynomials detect fibered 3-manifolds, arXiv:0805.1234

11. S. Friedl and S. Vidussi, A survey of twisted Alexander polynomials, Proc. of the Conf., The Mathematics of Knots: Theory and Application, in Heidelberg 2008.

12. H. Goda, T. Kitano and T. Morifuji, Reidemeister torsion, twisted Alexander polynomial and fibered knots, Comment. Math. Helv. 80 (2005), 51-61.

13. H. Goda and T. Morifuji, Twisted Alexander polynomial for $S L(2, \mathbb{C})$-representations and fibered knots, C. R. Math. Acad. Sci. Soc. R. Can. 25 (2003), 97-101.

14. J. Hillman, D. Silver and S. Williams, On reciprocality of twisted Alexander invariants, Algebr. Geom. Topol. 10 (2010), 1017-1026.

15. M. Hirasawa and K. Murasugi, Twisted Alexander polynomials of 2-bridge knots associated to metacyclic representations, arXiv:0903.0147

16. J. Hoste and P. D. Shanahan, A formula for the A-polynomial of twist knots, J. Knot Theory Ramifications 13 (2004), 193-209.

17. P. Kirk and C. Livingston, Twisted Alexander invariants, Reidemeister torsion, and CassonGordon invariants, Topology 38 (1999), 635-661.

18. T. Kitano, Twisted Alexander polynomial and Reidemeister torsion, Pacific J. Math. 174 (1996), 431-442. 
19. T. Kitano and T. Morifuji, Divisibility of twisted Alexander polynomials and fibered knots, Ann. Sc. Norm. Super. Pisa Cl. Sci. (5) 4 (2005), 179-186.

20. P. B. Kronheimer and T. S. Mrowka, Dehn surgery, the fundamental group and SU(2), Math. Res. Lett. 11 (2004), 741-754.

21. Thang T. Q. Le, Varieties of representations and their subvarieties of cohomology jumps for knot groups, Mat. Sb. 184 (1993), 57-82.

22. X. S. Lin, Representations of knot groups and twisted Alexander polynomials, Acta Math. Sin. (Engl. Ser.) 17 (2001), 361-380.

23. M. L. Macasieb, K. L. Petersen and R. M. Van Luijk, On character varieties of two-bridge knot groups, arXiv:0902.2195

24. T. Morifuji, Twisted Alexander polynomials of twist knots for nonabelian representations, Bull. Sci. Math. 132 (2008), 439-453.

25. K. Murasugi, On the genus of the alternating knot. I, II, J. Math. Soc. Japan 10 (1958), 94-105, 235-248.

26. K. Murasugi, On a certain subgroup of the group of an alternating link, Amer. J. Math. 85 (1963), 544-550.

27. F. Nagasato and Y. Yamaguchi, On the geometry of a certain slice of the character variety of a knot group, arXiv:0807.0714

28. T. Ohtsuki, Ideal points and incompressible surfaces in two-bridge knot complements, J. Math. Soc. Japan 46 (1994), 51-87.

29. T. Ohtsuki, R. Riley and M. Sakuma, Epimorphisms between 2-bridge link groups, in the Zieschang Gedenkschrift, Geometry and Topology Monographs 14 (2008), 417-450.

30. R. Riley, Nonabelian representations of 2-bridge knot groups, Quart. J. Math. Oxford Ser. (2) 35 (1984), 191-208.

31. M. Wada, Twisted Alexander polynomial for finitely presentable groups, Topology 33 (1994), $241-256$.

Department of Mathematics, Konkuk University, Seoul 143-701, Republic of Korea

E-mail address: tkim@konkuk.ac.kr

Department of Mathematics, Tokyo University of Agriculture and Technology, TOKYO 184-8588, JAPAN

E-mail address: morifuji@cc.tuat.ac.jp 